\title{
Selenium nanoparticles (SeNPs) have potent antitumor activity against prostate cancer cells through the upregulation of miR-16
}

\author{
Guolong Liao ${ }^{1 \dagger}$, Jiani Tang ${ }^{1 \dagger}$, Di Wang ${ }^{2}$, Haoru Zuo ${ }^{3}$, Qi Zhang ${ }^{3}$, Ying Liu ${ }^{3}$ and Haiyun Xiong ${ }^{1 *}$
}

\begin{abstract}
Objectives: This research aimed to examine the antitumor mechanisms of selenium nanoparticles (SeNPs) specifically against prostate cancers.

Methods: The antitumor activities of SeNPs against cancer cells were determined via MTT assay. The cell cycle was determined by detecting the DNA content, and apoptosis was determined via annexin V-Fluos staining kit. The microRNA expressions in cancer cells were analyzed via microarray and qRT-PCR. The potential targets of miR-16 were identified via luciferase analysis and mRNA expression determination. miR-16 functions in cancer cells were explored via the transient transfection of miR-16 mimic or inhibitor.

Results: SeNPs were most potent in prostate cancer cells, regardless of whether or not they were androgendependent. Furthermore, SeNP stimulation can induce cell cycle arrest and the apoptosis enhancement of prostate cancer cells. Microarray and molecular mechanism studies demonstrated that miR-16 could directly target cyclin D1 and BCL-2 to mediate SeNP apoptosis enhancement. Results show that the serum selenium levels positively correlate with miR-16 expressions, and they correlate with the overall and disease-free survival rates.
\end{abstract}

Conclusion: These results signify the cytotoxic potential of SeNPs in prostate cancer treatment.

Keywords: Selenium nanoparticles, Prostate cancer, Cell cycle, Apoptosis, MicroRNA

\section{Introduction}

Prostate cancer $(\mathrm{CaP})$ is the second most prevalent cancer globally [1]. Prostate cancer is also the fifth most common cancer-related cause of male death. Early-stage CaP requires androgens to grow, and if it is caught before progressing further, then it often responds well to androgen deficiency therapy [2]. However, advanced $\mathrm{CaP}$ can transform from androgen-dependent prostate cancer (ADPC)

\footnotetext{
*Correspondence: xiong_urology@sohu.com

${ }^{\dagger}$ Guolong Liao and Jiani Tang contributed equally to this work.

'Department of Urology, The Seventh Affiliated Hospital of Sun Yat-Sen University, Shenzhen 518107, China

Full list of author information is available at the end of the article
}

into androgen-independent prostate cancer (AIPC) [3]. Currently, no effective treatment exists for AIPC. Therefore, developing effective drugs for $\mathrm{CaP}$ is an urgent need, especially for AIPC, which could reduce the mortality and improve the quality of life of $\mathrm{CaP}$ patients.

Selenium has been shown to have antitumor properties, and selenium supplements have been used in individual anticancer therapies [4]. Owing to their minimal risk relative to selenium on its own, selenium nanoparticles (SeNPs) have had significant applications in medical diagnosis over the last decade, and thus, they have been widely used as dietary supplements and antioxidants [5]. In particular, SeNPs have occasionally been reported to 
exhibit antitumor effects against several tumor types, including glioma, lung cancer, and breast cancer $[4,5]$.

The promotion of apoptosis of cancer cells by SeNPs is generally believed to be an important mechanism in their inhibition of malignant tumors [4]. SeNPs have been found to regulate key apoptotic proteins, such as the caspase family, p53, and ROS [6]. However, these sporadic findings do not fully explain the antitumor functions of SeNPs. In particular, the question of whether or not microRNAs (miRNA), which play an important role in tumorigenesis and development, are regulated by SeNPs requires further study.

In the previous work of the authors, SeNPs were synthesized using new microbial technology and characterized via UV-Vis absorption spectroscopy [6]. In those studies, SeNPs were found to play an important role in inhibiting lung cancer cells but had little effect on normal cells. Similarly, SeNPs have been suggested to have potent cytotoxicity against tumor cells, but not against normal cells, in studies of cervical carcinoma [7], hepatocarcinoma [8], and colorectal cancer [9]. Therefore, plans were made to test for the inhibitory effects of SeNPs on other cancer cells and to explore their molecular mechanisms. Preliminary experiments confirmed that SeNPs are significantly active on several cancer cell lines and that their activity on $\mathrm{CaP}$ cells is stronger than on other cancer cells. This indicates that SeNPs might be most effective in $\mathrm{CaP}$ and that SeNPs lead to cell cycle arrest and apoptosis enhancement in various $\mathrm{CaP}$ cell lines and primary isolated tumor cells. Molecular biological experiments suggest that SeNPinduced apoptosis enhancement is achieved in part by miR-16 upregulation.

\section{Material and methods}

\section{Preparation and characterization of SeNPs}

The methods of the SeNPs biosynthesis and characterization were described in our previous work [6]. Briefly, a 250-ml Erlenmeyer flask was used to harvest the bacterial seed culture in $100 \mathrm{ml} \mathrm{N}$-broth growth medium and incubated at $30^{\circ} \mathrm{C}$ for about $24 \mathrm{~h}$. Three millimolars of sodium selenite $(0.0518 \mathrm{~g})$ and activated Escherichia coli (E. coli) culture (1 $\mathrm{ml}$ ) were inoculated into $100 \mathrm{ml}$ sterilized N-broth medium, placed in an incubator at $30^{\circ} \mathrm{C}$ for $48 \mathrm{~h}$ at $150 \mathrm{rpm}$. UVvisible (HACH, DR 5000, USA) spectrophotometer was employed to analyze the samples. The mixture was centrifuged at $12,000 \mathrm{rpm}$ for $10 \mathrm{~min}$ to isolate the particles from the reaction mixture and then washed thrice using acetone and distilled water. For control experiment, active E. coli culture was autoclaved for $15 \mathrm{~min}$ and cultivated into $100 \mathrm{ml}$ sterilized N-broth medium with $3 \mathrm{mM}(0.0518 \mathrm{~g})$ sodium selenite under similar conditions as described above. For characterization of SeNPs, transmission electron microscopy (TEM; Zeiss-EM10C) was employed to study the dimension and morphology of the synthesized SeNPs at a raising voltage of $80 \mathrm{kv}$. Ultraviolet visible (UV-vis) spectrophotometer at a resolution of $2 \mathrm{~nm}$ was used to record the absorption spectra at a wave length range of $200-1000 \mathrm{~nm}$. For the elemental analysis of the product, energy-dispersive X-ray analysis (EDAX) analyzer (JSM-7600F, JEOL, Japan) was used. XRD analysis (Xpert pro, PANalytical, Holland) was performed to investigate the crystalline phase of synthesized SeNPs.

\section{Cell line culture}

Eight tumor cell lines, including Colon-26 (colon cancer), LNCaP (CaP), HepG2 (liver cancer), Hela (cervical cancer), A549 (NSCLC), MCF7 (breast cancer), A498 (renal cancer), and WM-115 (melanoma), which were purchased from ATCC (Manassas, VA, USA), were used in the preliminary screening of the anticancer activity of SeNPs. For further study on the antitumor activity of SeNPs against CaP, LNCaP (ADPC), PC-3 (AIPC), C4-2 (AIPC), LNCaP-A (AIPC), PWR-1E (normal prostate), and RWPE-1 (normal prostate), also purchased from ATCC, were included. For the cell culture, $10 \%$ fetal bovine serum (FBS) (Invitrogen, Grand Island, NY, USA), $100 \mathrm{U} / \mathrm{ml}$ streptomycin, and $100 \mathrm{U} / \mathrm{ml}$ penicillin (Hyclone laboratories Inc., South, UT, USA) were added to Roswell Park Memorial Institute (RPMI-1640) to prepare the cell culture medium. The culture environment was $5 \%$ carbon dioxide and $37^{\circ} \mathrm{C}$ with humidified air in an incubator (Thermo Fisher, Waltham, MA, USA).

\section{Clinical samples}

This study was conducted in the urology department of the Seventh Affiliated Hospital of Sun Yat-Sen University. One hundred seventeen inpatients with $\mathrm{CaP}$ and 33 healthy controls were enrolled in this study. Serum samples from all patients, and their matched controls, were collected during morning admission (on an empty stomach). Patients treated for $\mathrm{CaP}$ before their serum sample extraction were excluded from this study. ADPC patients or AIPC patients were considered by referring to the documentary reporting criterion [10]. Sixty-two patients were allocated to the AIPC group and while others were allocated to the ADPC cases. The serum selenium level was determined via graphite Atomic Absorption Spectrophotometer (AAS) as described in the previous report [11].

\section{Isolation of the primary CaP cells}

$\mathrm{CaP}$ tissues and adjacent normal tissues were obtained from $117 \mathrm{CaP}$ patients via surgery, then primary cultured cells were extracted from them according to previous reports [12]. The CaP tissues and adjacent normal tissues were washed with $95 \sim 100 \%(\mathrm{v} / \mathrm{v})$ ethanol and PBS (0.01M, $\rho H 7.4)$, then they were placed in a sterile Petri dish containing pre-cooled PBS. Residual fat was removed with anatomical forceps and scissors. Next, 
$0.25 \%$ trypsin or $2000 \mathrm{U} / \mathrm{ml}$ collagenase was added into the dish, digestion in a $37^{\circ} \mathrm{C}$ water bath for $30 \mathrm{~min}$ was conducted, the digestive juice was removed, washing was done three times with washing solution, the medium was washed once, suspended with complete medium, and a straw was used to blow and disperse into the cell suspension. Then, the mixture was centrifuged, and the pellet was re-suspended in PBS containing 0.25\% tryp$\sin / 1 \mathrm{mM}$ EDTA for $30 \mathrm{~min}$ at $37^{\circ} \mathrm{C}$. The trypsin is neutralized with $10 \% \mathrm{FCS}$, and the cells were washed twice in PBS. The cells are subsequently passed through a cell strainer to ensure single-cell suspension (BD Falcon, NJ). The cells were cultured via RPMI-1640 medium.

\section{MTT assay}

The antitumor activities of SeNPs against different types of cancer cells were determined via MTT (3-(4,5-dimethylthiazole-2-yl)-2,5-diphenyltetrazolium bromide). After counting the cells to be measured, the cells were inoculated into 96well plates with 10,000 cells per well for $24 \mathrm{~h}$, then the cells were treated with SeNPs at certain concentrations for $24 \mathrm{~h}$. Subsequently, the cells were treated with MTT solution for $3 \mathrm{~h}$, DMSO was added, then the cells were placed for $15 \mathrm{~min}$. Finally, an ELISA microplate reader (DYNEX, USA) was used to measure absorbance at $570 \mathrm{~nm}$ (OD value). The standard MTT assay curve was established via the following process: Cells were counted then inoculated into clear cell culture plates at seven concentrations: 1000/well, 2000/well, 4000/well, 8000/well, 16000/well, 32000/well, and 64000/ well. They were incubated with MTT reagent at $37^{\circ} \mathrm{C}$ for 3 h. After incubation, the cells were treated with DMSO at room temperature for $15 \mathrm{~min}$. Absorbance was measured at $\mathrm{OD}=570 \mathrm{~nm}$, and a standard curve was drawn based on the correlation between OD values and cell numbers. The cell number was calculated via the corresponding OD value through the standard curve. The SeNP inhibition rate was calculated via the following equation: inhibition rate $=($ cell number(control) - cell number(SeNP treatment))/cell number(control) $\times 100 \%$.

\section{Cell cycle analysis}

Cells were cultured in a serum starvation medium at the G0 stage for 3 days, and then, the medium was replaced by a medium containing $10 \%$ FBS for the culture. Cell cycle progression is monitored by detecting the DNA content [13]. Cells were washed twice with PBS, trypsinized, and then fixed in $70 \%$ methanol for $2 \mathrm{~h}$ at $-20^{\circ} \mathrm{C}$. Subsequently, cells were precipitated ( $5 \mathrm{~min}$ of centrifugation at $500 \times g$ at $4{ }^{\circ} \mathrm{C}$ in a Sigma $6 \mathrm{~K} 15$ centrifuge), washed with PBS, and re-suspended in $1 \mathrm{ml}$ of PBS containing $40 \mathrm{U}$ of RNase A per $\mathrm{ml}$ and $40 \mu \mathrm{g}$ of propidium iodide per milliliter. After incubating for $30 \mathrm{~min}$ at $37^{\circ} \mathrm{C}$, DNA flow cytometric analysis was performed with an EPICS XL-MCL flow cytometer (Coulter). Multicycle
AV software (Phoenix Flow Systems) was used for quantification.

\section{Cell apoptosis analysis}

Apoptotic cells were detected via annexin V-Fluos staining kit (Roche-Boehringer). Cells $\left(1 \times 10^{6}\right) /$ well were plated in 6-well plates for $24 \mathrm{~h}$ then treated with SeNPs at specific concentrations for an additional $24 \mathrm{~h}$ in a $5 \% \mathrm{CO}_{2}$ humidified atmosphere at $37^{\circ} \mathrm{C}$. At the end of the incubation period, the treated cells and controls were harvested and incubated with Annexin V and PI for 15 min before being analyzed on a flow cytometer with $488 \mathrm{~nm}$ excitation, 515 $\mathrm{nm}$ for Annexin $\mathrm{V}$ detection, and a filter with the wavelengths above $600 \mathrm{~nm}$ for PI detection.

\section{Determination of Caspase-3 activity}

The flourometric method was used to evaluate the Caspase- 3 activity according to the previous report [6]. Twelve-well plates were seeded with cells and incubated for $24 \mathrm{~h}$. Later, the cells were treated with SeNPs and incubated for $24 \mathrm{~h}$. A lysis buffer was used for cell lysis after harvesting, then the cells were incubated for $1 \mathrm{~h}$ in ice. Caspase-3 substrates (Ac-DEVD-AMC) were used to determine the Caspase activity by measuring fluorescence intensity at an excitation wavelength of $380 \mathrm{~nm}$ and $460 \mathrm{~nm}$ as an emission wavelength.

\section{Reverse transcription-quantitative polymerase chain reaction ( $R T-q P C R)$ assay}

TRIzol reagent (Invitrogen, Grand Island, NY, USA) was used to obtain the total RNA from cells. Subsequently, qRT-PCR for mRNA was performed via the PrimeScript RT-PCR kit (Takara, Bio, Inc., Shiga, Japan) on an IQ5 fluorescence quantitative PCR detector (Bio-Rad, Hercules, CA, USA). Primer sequences for all the targets and $\beta$-actin are described in the following way: Primer for $\beta$-actin: forward 5'-AAGGAAGCTTGGCG TTGTGA-3'; reverse: 5'-GAGAGGTGAGGAGTCTTA TG-3'. Primer for P21: forward 5'-TAGCAGCGGA ACAAGGAG-3'; reverse: 5'-AAACGGGAACCAGG ACAC-3'. Primer for cyclin D1: forward 5'-GTCTTC CCGCTGGCCATGAACTAC-3'; reverse: 5'-GGAAGC GTGTGAGGCGGTAGTAGG-3'. Primer for BAX: forward 5'-TCCACCAAGAAGCTGAGCGAG-3'; reverse: 5'-GTCCAGCCCATGATGGTTCT-3'. Primer for BCL2: forward $5^{\prime}$-TTCTTTGAGTTCGGTGGGGTC-3'; reverse: $5^{\prime}$-TGCATATTTGTTTGGGGCAGG-3'. The following parameters were used for the reverse transcription reaction: $65^{\circ} \mathrm{C}$ for $5 \mathrm{~min}, 37^{\circ} \mathrm{C}$ for $15 \mathrm{~min}$, and $98^{\circ} \mathrm{C}$ for $5 \mathrm{~min}$. The following parameters were used for the subsequent PCR reaction: $95^{\circ} \mathrm{C}$ for $30 \mathrm{~s}$ and then 40 cycles of $95^{\circ} \mathrm{C}$ for $5 \mathrm{~s}, 60^{\circ} \mathrm{C}$ for $5 \mathrm{~s}$, and $72{ }^{\circ} \mathrm{C}$ for $30 \mathrm{~s}$.

qRT-PCR for microRNAs was conducted using TaqMan miRNA assays (Ambion; Thermo Fisher Scientific, Inc.) 
and has-miR-16 RT-PCR primer set (Abbexa, Inc., Cambridge, UK). Reverse transcription reactions were performed via $\mathrm{TAQMAN}^{\circ}$ microRNA RT kit (Ambion; Thermo Fisher Scientific, Inc.) under the following conditions: $16^{\circ} \mathrm{C}$ for $30 \mathrm{~min}, 42{ }^{\circ} \mathrm{C}$ for $30 \mathrm{~min}$, and $84^{\circ} \mathrm{C}$ for 5 min. PCR reactions were conducted under the following conditions: $95^{\circ} \mathrm{C}$ for $2 \mathrm{~min}$ followed by 40 cycles of $95^{\circ} \mathrm{C}$ for $15 \mathrm{~s}$ and $60^{\circ} \mathrm{C}$ for $30 \mathrm{~s}$. U6 small nuclear RNA was used as an endogenous control for data normalization.

\section{Microarray analysis of miRNA expression}

Microarray detection was carried out via the Agilent Human miRNA microarray platform (Agilent Technologies) by Shanghai Biochip Co., Ltd. (Shanghai, China). The Agilent human microarray platform (Agilent technology) and its microarray scanner were used for microarray analysis and microarray slide scanning. The scanned image was analyzed via Feature Extraction software 10.7.1.1 (Agilent). Genespring 12.0 (Agilent) was used to normalize the raw data.

\section{Dual luciferase assay for BCL-2 and cyclin D1 targets identification}

Double-stranded DNA fragments containing the potential miR-16 binding site sequences of BCL-2 and cyclin D1 genes were prepared. Then, the DNA fragments were introduced into the pGL3-promoter vector (Promega, Madison, WI, USA) to construct pGL3-wide type-BCL-2 and pGL3-wide type-cyclin D1 plasmid. Both pGL3-wide

A

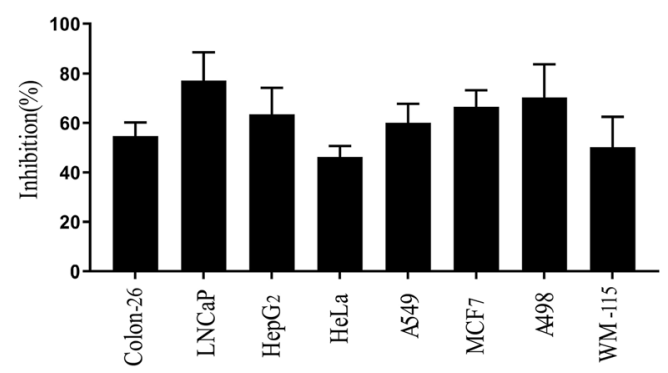

B
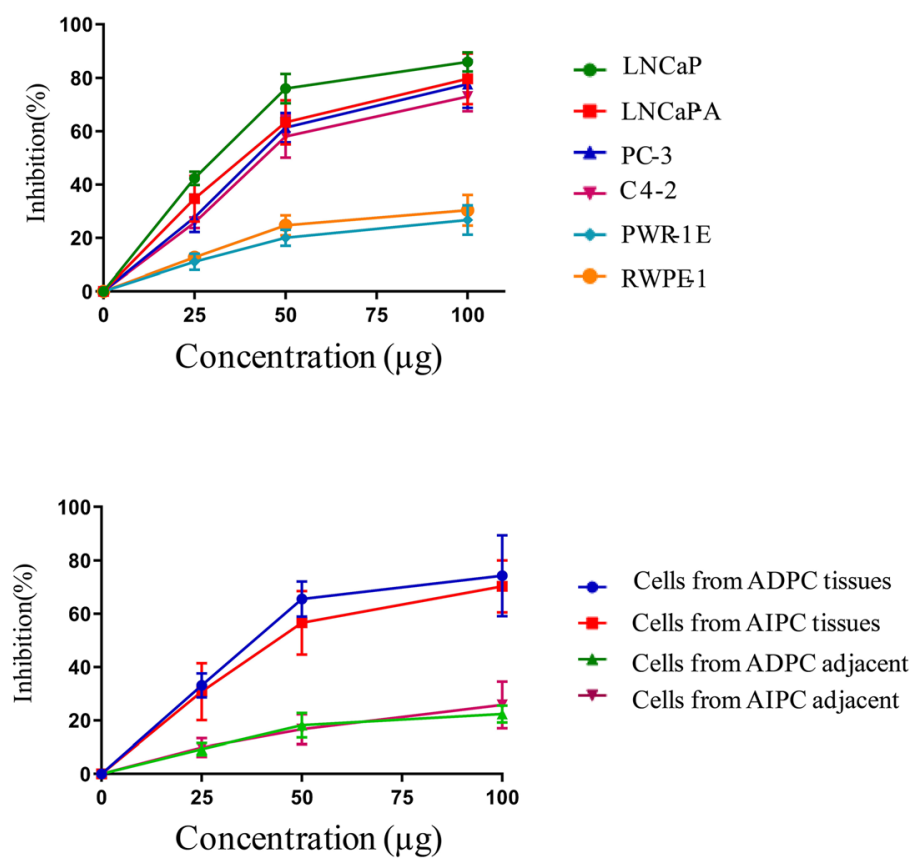

Fig. 1 SeNPs has potent antitumor activities against different cancer cells in vitro. a Inhibition percentages expressed by the SeNPs against eight cancer cell line at a single-dose of $50 \mu \mathrm{g}$. $\mathbf{b}$ Inhibition percentages expressed by the SeNPs against four prostate cancer cell lines and two normal prostate cells at dose ranges from 25 to $50 \mu \mathrm{g}$. $\mathbf{c}$ Inhibition percentages expressed by the SeNPs against patients-derived prostate cancer cells at dose ranges from 25 to $100 \mu \mathrm{g}$. The \%inhibition values were calculated by subtracting the growth percentages from 100 . 
type-BCL-2 and pGL3-wide type-cyclin D1 had GCUGCU sequence in the binding site for miR-16. By using a site-directed mutagenesis kit (Stratagene), this GCUGCU sequence was replaced with GAAAAU to construct pGL3-mutant-BCL-2 and pGL3-mutant-cyclin D1. The recombinant plasmid constructions were identified via DNA sequencing.

\section{Statistical analyses}

By using SPSS software version 17.0 (SPSS Company, Chicago, Illinois, USA), the statistical differences between the experimental group and the control group were determined via the $\mathrm{t}$-test of the double-tailed Student. All experiments in this study were repeated three times, and data are represented as the mean \pm standard deviation. If the $p$ value is less than 0.05 , a statistical difference between the two comparison groups will be set.

\section{Results}

In vitro antitumor activity of SeNPs against eight tumor cell lines

The essential properties of nanoparticles, including element composition, size, shape composition, and surface structure, can affect the anticancer activity of nanoparticles [14]. The average SeNP crystalline size used in this study was calculated as $88.89 \mathrm{~nm}$ via X-ray diffraction, which was identified as a hexagonal ring structure with a diffraction ring pattern [6]. The antitumor activities of this kind of SeNPs against eight cancer cell lines were initially tested. Figure 1a shows the inhibition
A

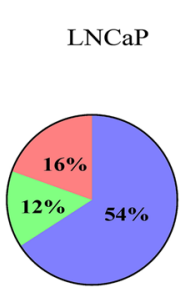

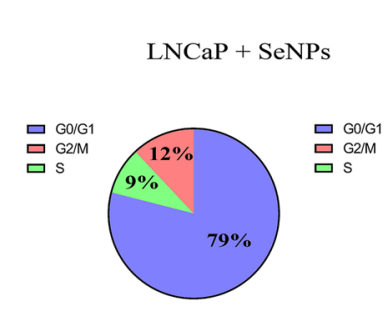

C

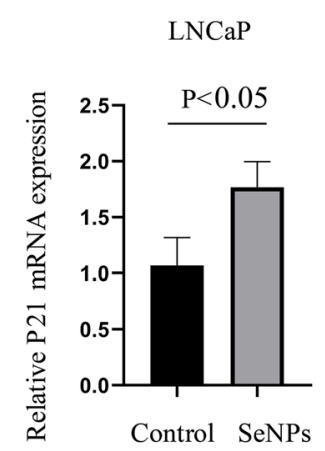

E

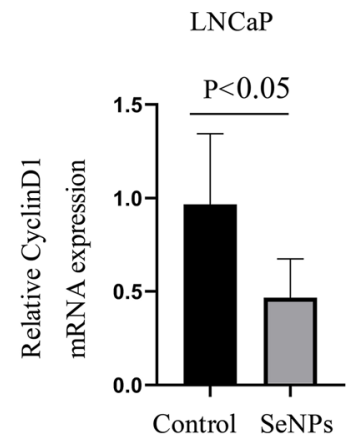

B

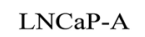

LNCaP-A + SeNPs
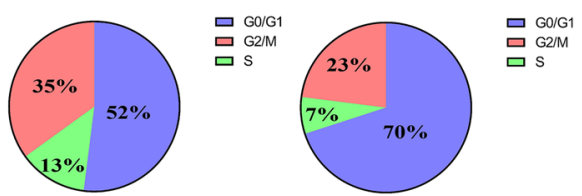

D

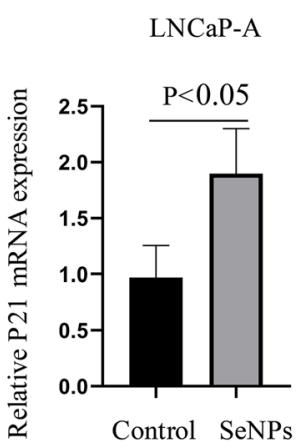

F

LNCaP-A

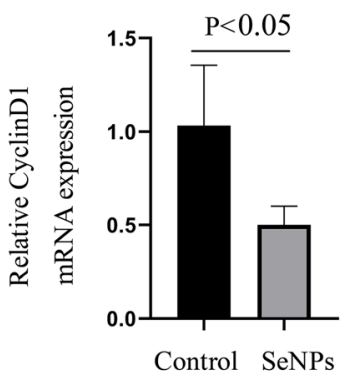

Fig. 2 SeNP treatment lead to cell cycle arrest in LNCaP and LNCaP-A cells. a, b Cell cycle analysis in a LNCaP and $\mathbf{b} L N C a P-A$ after SeNP treatment. $\mathbf{c}$, d Expression of p21 (CDKN1A, c) in $\mathbf{c} L N C a P$ and $\mathbf{d} L N C a P-A$ after SeNP treatment. e, $\mathbf{f}$ Expression of cyclin D1 in a LNCaP and $\mathbf{b}$ LNCaP-A after SeNP treatment. SeNP dose: $50 \mu \mathrm{g}$ 
percentages caused by a single $50 \mu \mathrm{g}$ dose of SeNPs. The inhibition percentages of SeNPs on cancer cells ranged from $46.3 \pm 4.4 \%$ to $77.2 \pm 11.4 \%$. The most obvious inhibitory effect was observed in CaP cells.

\section{In vitro antitumor activity of SeNPs against the CaP cells}

Subsequently, the anticancer activity of SeNPs at different concentrations was measured against several $\mathrm{CaP}$ cell lines, including ADPC and AIPC. These results show that the treatment of LNCaP cells with $25 \mu \mathrm{g} / \mathrm{ml}, 50 \mu \mathrm{g} / \mathrm{ml}$, and $100 \mu \mathrm{g} / \mathrm{ml}$ each led to inhibition percentages of $42.3 \pm 2.5 \%$, $77.2 \pm 11.4 \%$, and $82.7 \pm 10.5 \%$. AIPC cell inhibition was slightly lower, especially with a low concentration of SeNPs. SeNPs had no significant effect on normal healthy cells (Fig. 1b). For each $\mathrm{CaP}$ patient, primary cells were extracted from $\mathrm{CaP}$ tissues and tumor-adjacent tissues then the inhibitory effect of SeNPs against these cells was measured. Figure 1c shows that SeNPs have potent toxicity against both ADPC and AIPC the primary isolated CaP cells of ADPC and AIPC. Collectively, these results indicate that SeNPs have a strong cytotoxic effect against $\mathrm{CaP}$ cells.
SeNP treatment leads to cell cycle arrest and enhanced apoptosis in CaP cells

Next, the anti-CaP mechanism of SeNPs in LNCaP and LNCaP-A cells was studied. When compared with the control group, the cell cycle arrest rate in $\mathrm{LNCaP}$ and LNCaP-A cells was significantly increased after treatment with $50 \mathrm{ug} / \mathrm{ml}$ SeNPs (Fig. 2a, b). Cell cycle arrest has been reported to be induced by $\mathrm{p} 21$ and inhibited by cyclin D1 [12]. As expected, the p21 mRNA expression was significantly increased in SeNP-treated LNCaP and LNCaP-A cells (Fig. 2c, d), while the cyclin D1 expression was decreased (Fig. 2e, f).

Furthermore, the SeNP treatment was found to significantly enhance both the apoptosis rate (Fig. 3a, b) and caspase 3 activity (Fig. 3c, d) in LNCaP and LNCaP-A cells. In addition, $24 \mathrm{~h}$ after SeNP treatment, the BAX mRNA levels were upregulated (proapoptotic, Fig. 3e, f), and the BCL-2 expression was significantly downregulated (anti-apoptotic, Fig. 3g, h). These results lead to the conclusion that SeNP treatment can cause cell cycle arrest and apoptosis enhancement.

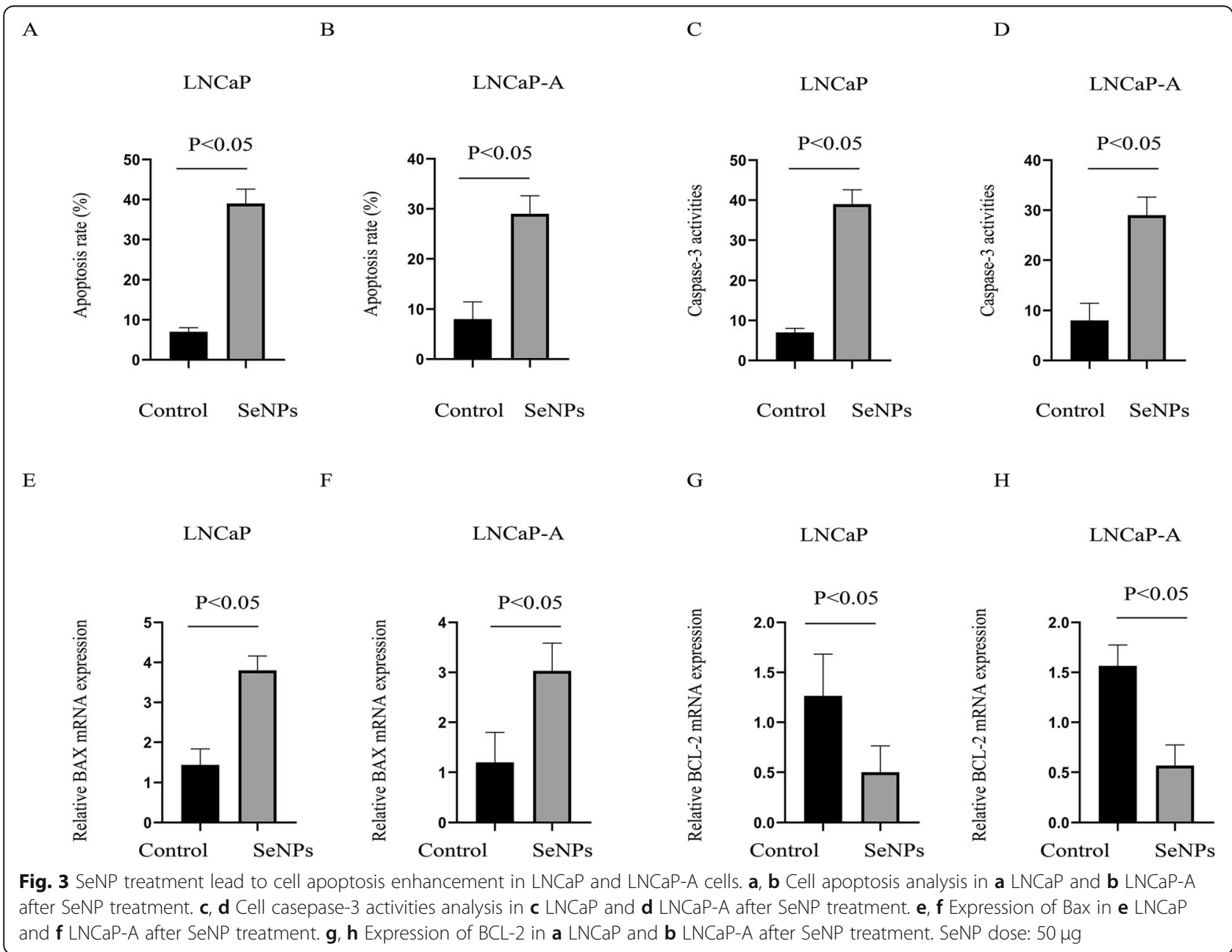


Global changes in the expression of miRNAs in CaP cells during SeNP treatment

SeNP-treated LNCaP and LNCaP-A cells were used to investigate global changes in the miRNA expressions. With a cutoff line of $>1.5$ fold change and $p \leq 0.05$ (identified by oneway ANOVA), 212 genes in $\mathrm{LnCaP}$ and 244 genes in LnCaP-A were differentially expressed after SeNP treatment. Venn analysis showed that a total of 16 differentially regulated genes overlapped in SeNP-treated LNCaP and LNCaPA cells (Fig. 4a). Figure 4b shows the clustering of these genes. Among these genes, miR-16 showed the largest expression change in a microarray assay (Fig. 4c). The miR-16 expression profile in SeNP-treated LNCaP and LNCaP-A cells was further validated via qPCR analysis (Fig. 4d, e).

\section{SeNP treatment enhances cell apoptosis in CaP cells via miR-16 upregulation}

TargetScan analyses (http://www.targetscan.org/) were used to find that cyclin D1 and BCL-2, key proteins in the proliferation and apoptosis of cancer cells, might bind with miR-16 at a GCUGCU sequence site (Fig. 5a). This prediction was confirmed using a luciferase report system, which showed that miR-16 mimics significantly inhibited luciferase activity in HEK293 cells containing the wild-type 3'UTR of BCL-2 or cyclin D1. However, luciferase activity was not affected in cells with the mutant 3'UTR of BCL-2 or cyclin D1 (Fig. 5b). Moreover, miR-16 mimics significantly decreased the protein expression of BCL-2 and cyclin D1 in LNCaP and LNCaPA cells (Fig. 5c, d), indicating that miR-16 upregulation might be essential to mediating SeNP antitumor activity. To investigate this point, LNCaP and LNCaP-A cells were transiently transfected with a miR-16 inhibitor in conjunction with a treatment of $50 \mu \mathrm{g} / \mathrm{ml}$ SeNPs. Figures $5 \mathrm{e}$ and $\mathrm{f}$ show the use of an miR-16 inhibitor sharply reversed the impacts of SeNPs on the apoptosis rate. These findings suggest that SeNP treatment increases apoptosis rates by enhancing miR-16 expression.

\section{Selenium content in CaP patients is correlated with clinical prognosis}

Next, whether or not the role of selenium in patients was consistent with the results needed to be further verified. In the entire patient cohort, the serum selenium concentration for $\mathrm{CaP}$ patients before treatment was significantly lower than that for healthy controls (Fig. 6a). A positive correlation existed between selenium content and serum miR-16 expression, which was in agreement with the cytological experiment results (Fig. 6b). Subsequently, all $\mathrm{CaP}$ subjects were categorized into either

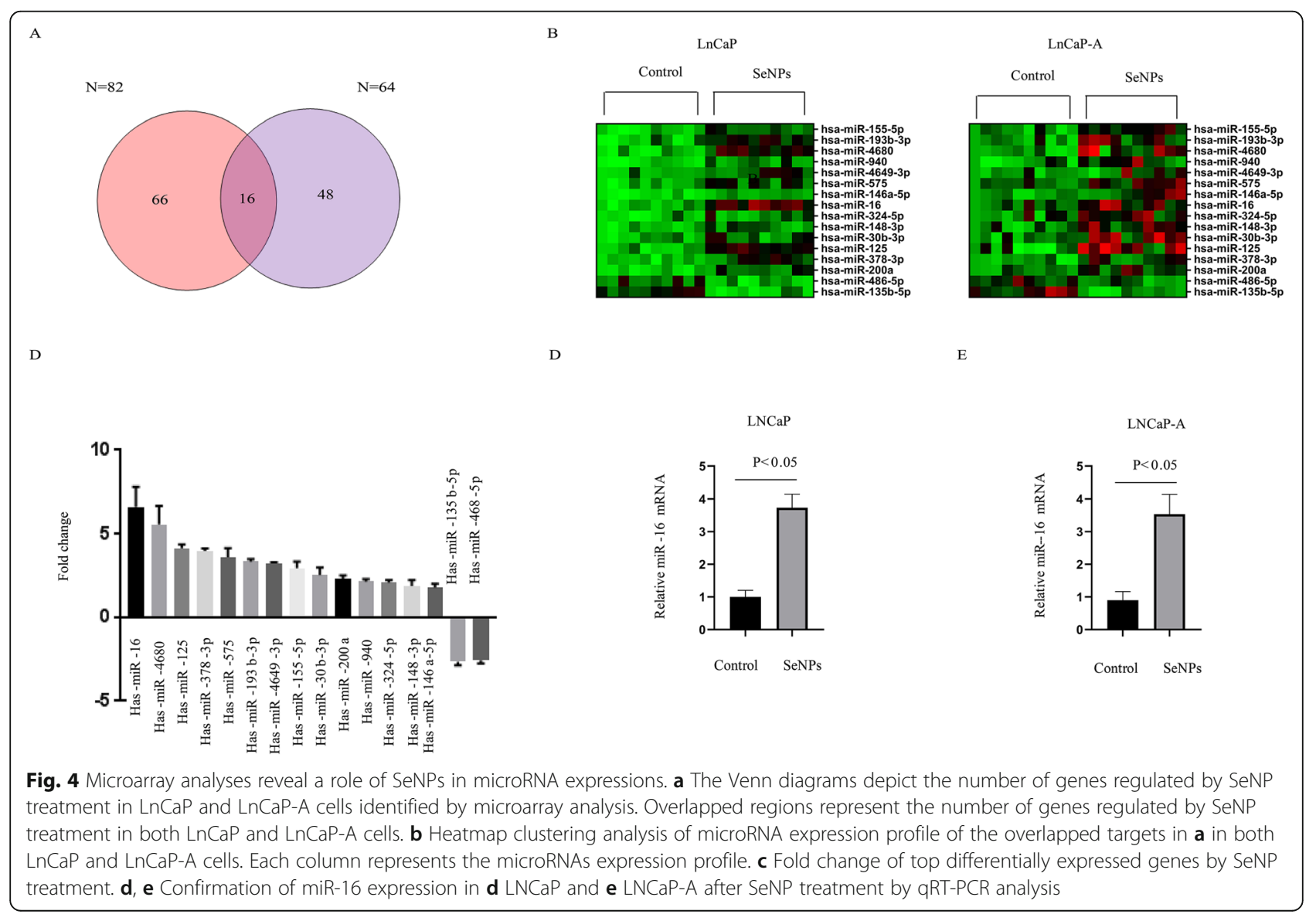


A

BCL -2

$$
\begin{aligned}
& \text { BCL-2 mutant 5' ...GAAUAUCCAAUCCUGUGAAAAUA... } \\
& \text { BCL-2 wide type } 5^{\prime} \text {...GAAUAUCCAAUCCUGUGCUGCUA... } \\
& \text { II I I | II | || | } \\
& \text { miR-16 3' GCGGUUAUAAAUGC ----ACGACGAU } \\
& \text { Cyclin D } 1 \\
& \text { Cyclin D1 mutant } \quad 5 \text { '.CCAUUUUCUUAUUGC ----GAAAAUAC... } \\
& \text { Cyclin D1 wide type 5'.CCAUUUUCUUAUUGC ----GCUGCUAC... } \\
& \text { miR-16 3' GCGGUUAUAAAUGCACGACGAU }
\end{aligned}
$$

C

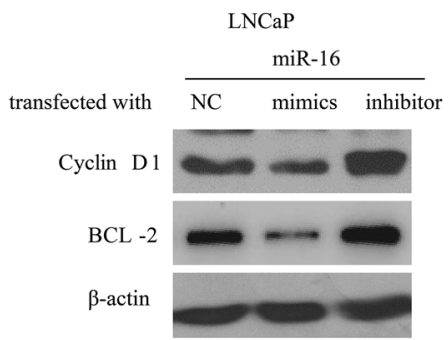

E

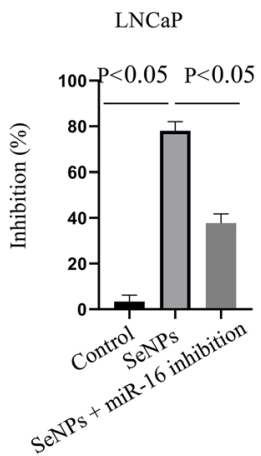

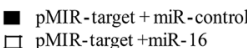

pMIR-target + miR-16

pMIR-mutant-target +miR-16
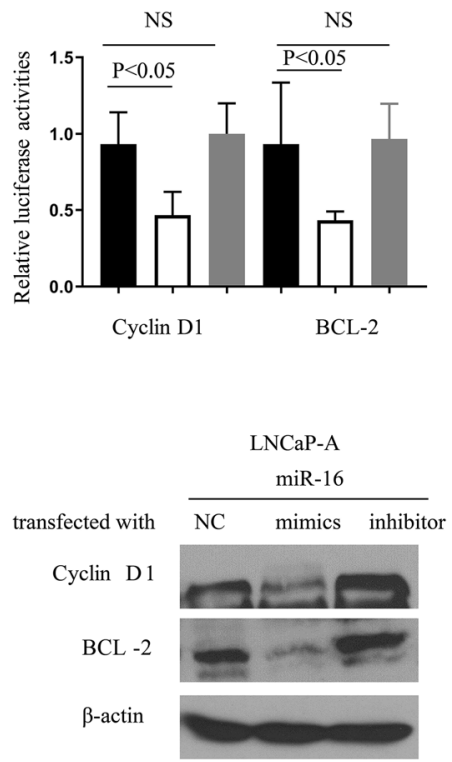

$\mathrm{F}$

LNCaP-A

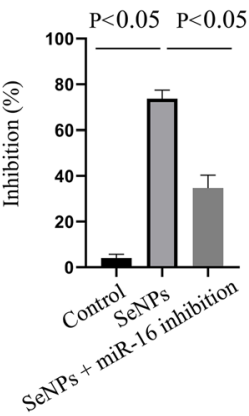

Fig. 5 SeNP treatment enhance the cell apoptosis of the prostate cancer cells through miR-16 upregulation. a Sequence alignment of miR-16 and its target sites in 3' untranslated regions of BCL-2 and cyclin D1. BCL-2 and cyclin D1 mutant site was shown in red. b HEK293 cells were transiently co-transfected with luciferase report vectors, and either miR-16 mimics or scrambled miR-control. Luciferase activities were normalized to the activity of Renilla luciferase. $\mathbf{c} L N C a P$ and $\mathbf{d}$ LNCaP-A were transient transfected with miR-16 inhibitor, together with SeNP treatment, and the protein expressions of $B C L-2$ and cyclin D1 were determined. e LNCaP and $\mathbf{f} L N C a P-A$ were transient transfected with miR-16 inhibitor, together with SeNP treatment, and the inhibition percentages expressed by the SeNPs were determined. The \%inhibition values were calculated by subtracting the growth percentages from 100. SeNP dose: $50 \mu \mathrm{g}$

high or low serum selenium groups according to whether or not they were above or below the mean value and were subsequently followed up $(57.1 \mu \mathrm{g} / \mathrm{L})$. KaplanMeier analysis showed that patients in the high selenium cohort had longer overall survival (OS) time and disease-free survival (DSF) time (Fig. 6c, d). In summary, the data from these studies suggest that higher selenium concentrations in $\mathrm{CaP}$ patients before treatment correlates with better prognoses.

\section{Discussion}

SeNPs have been suggested to have antitumor activity, and many studies have shown that they have high specific toxicity for cancer cells [15-17]. However, to date, whether or not SeNPs have different inhibitory potential against different types of tumors has not been made clear. This study explored SeNP toxicity on eight cancer cell lines, and SeNPs were found to have the strongest effect on CaP. Several previous reports have suggested 
A

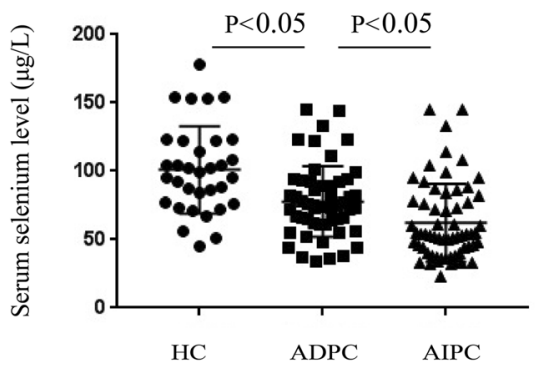

$\mathrm{C}$

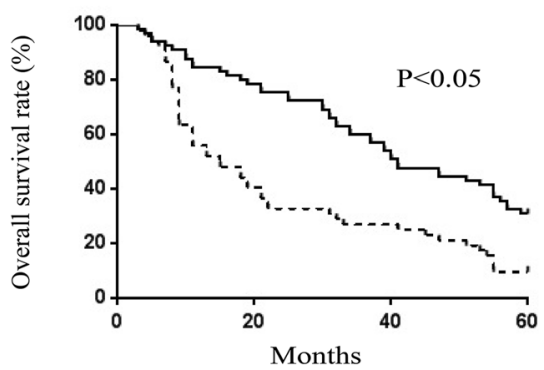

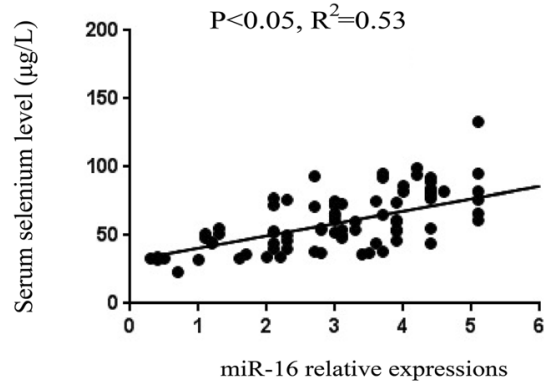

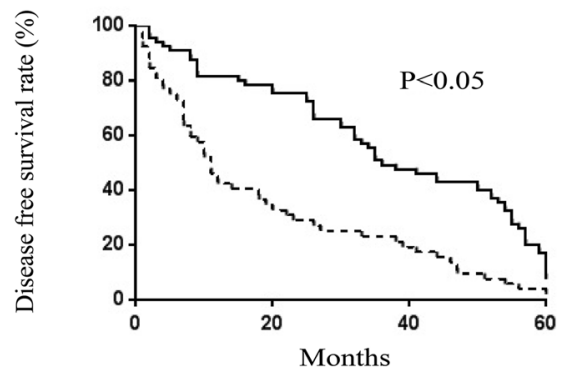

Fig. 6 Serum selenium content in prostate cancer patients is correlated with clinical prognosis. a Comparing the serum selenium content among AIPC, ADPC, and healthy control groups. $\mathbf{b}$ Determination of the correlation between serum selenium content and serum miR-16 expressions. The c overall survival and $\mathbf{d}$ disease-free survival of prostate cancer patients with different serum selenium level. Solid line: serum selenium level high group; dotted line: serum selenium level low group

that SeNPs exhibit strong inhibitory effects on CaP $[18$, 19]. For example, Sonkusre et al. reported that SeNPs have potent toxicity against PC-3 cancer cells [18]. Kong et al. demonstrated that SeNPs could suppress the growth of LNCaP cancer cells [19]. However, these studies primarily focused on a single $\mathrm{CaP}$ cell line. Furthermore, the clinical data on SeNP activity is sparse. This work appears to be a very early exploration of the antitumor effect of SeNPs against patient-derived CaP cells in vitro. SeNP inhibitory potential was slightly less for primary isolated $\mathrm{CaP}$ cells, as compared with $\mathrm{CaP}$ cell lines. This is possibly due to the existence of nonneoplastic cells in primary isolated cells. However, these experiments generally confirm that SeNPs have a significant inhibitory effect on patient-derived $\mathrm{CaP}$ cells, regardless of whether or not they were androgendependent. Meanwhile, SeNP toxicity on normal prostate cells is relatively low. This data indicates that SeNPs could represent a promising anti-CaP drug.

The molecular mechanism by which SeNPs induce tumor suppression is not yet fully understood. SeNPs are generally believed to be able to trigger tumor cell apoptosis by enhancing cellular uptake and blocking reactive oxygen species (ROS) [20]. Huang et al. have shown that SeNPs can stimulate cancer cell autophagy, thereby playing an anticancer role [21]. The report by Sonkusre showed that SeNPs induced TNF upregulation, which can activate cancer cell necrosis [18]. Vekariya et al. showed that SeNPs inhibited cell growth and the synthesis of DNA, RNA, and proteins, suggesting that SeNPs may alter the expression of a large number of functional molecules including non-coding RNA [17].

Additional evidence regarding the novel role of miRNAs in the cell cycle and apoptosis of cancer cells has been found recently. Most miRNA functions through the suppression of their target genes [22-24]. Unexpectedly, few reports have come up describing the relationship between SeNP treatment and miRNA expression. The highlight of this study was that using microarray technology, a series of microRNAs were found closely related to tumor development, such as miR-30b [25], miR155 [26], and miR-16 [27, 28]. miR-30b has been shown to inhibit tumor cell proliferation, invasion, and metastasis [25]. miR-155 was also reported to be a tumor suppressor activated via the targeting of many key proteins [26]. Together with previous reports, the data gathered in this report indicates that SeNP-regulated microRNAs likely play an important role in mediating SeNP functions. Among the identified miRNAs, miR-16 was measured as the most dramatically different between the SeNP treatment group 
and the control group. miR-16 has been reported as highly related to cell cycle and apoptosis regulation [27, 28]. These results demonstrated that SeNPs upregulate miR16, therefore downregulating two key targets of miR-16: cyclin D1 and BCL-2. This may be the direct route through which SeNPs promote cancer cell apoptosis. The miR-16 level in seven other tumor cell lines, including Colon-26, HepG2, Hela, A549, MCF7, A498, and WM-115, was measured, and it was found that SeNPs can also upregulate miR-16 expression in these cells (data not shown). Considering that miR-16 can regulate tumor development in a variety of tumor types [29], we conclude that the miR-16 upregulation by SeNPs may be a common anticancer mechanism in a variety of tumor cells.

SeNP antitumor effects against CaP led us to consider whether selenium levels in patients could affect tumor prognoses. A positive correlation between serum selenium levels and miR-16 expression was found. Furthermore, higher selenium concentrations were associated with better prognoses, confirming the trends measured in the in vitro assays of this study.

One limitation of this article is that the activity of SeNPs against $\mathrm{CaP}$ in animal models has yet to be confirmed. However, this work is already underway. The goal of these follow-up animal experiments is to test the toxicity and pharmacokinetic profile of SeNPs. Some earlier publications offer inspiration in this direction of study: Shahverdi et al. found that SeNPs had low toxicity and potent activity in a mouse model of breast cancer [30]. Meanwhile, Nazıroğlu et al. summarized their recent in vivo studies on animals treated with intraperitoneal or oral nanoparticles, where they found that nanoparticles, including SeNPs, can accumulate around tumors and cause direct cytotoxic effects on tumor cells [31]. These results encourage us to further explore the cytotoxic effects of SeNPs against CaP in vivo. Another limitation of this study is that it cannot be proven conclusively whether SeNPs only work through miR-16, or whether other mechanisms in mediating SeNPs functions and future work on the application of selenium nanoparticles, are to be expected.

\section{Conclusion}

Taken together, these results have evidently signified that SeNPs have strong killing activity against $\mathrm{CaP}$ cells, and high selenium content in $\mathrm{CaP}$ patients may indicate a better prognosis.

\section{Acknowledgements}

We would like to thank Editorbar (https://www.editorbar.com/) for English language editing.

\section{Authors' contributions}

$H X, G L$, and JT performed the cell-based experiments and wrote this part into the manuscript. DW prepared and characterized the SeNPs. HZ, $\mathrm{QZ}$, and $\mathrm{YL}$ performed the clinical data-based experiments and wrote this part into the manuscript. All authors read and approved the final manuscript.

\section{Funding}

This work was financially supported by the Research Project of the Chinese Medicine Science and Research Foundation of the Health Bureau of Shenzhen Guangming District (GM2019020021).

\section{Availability of data and materials}

The datasets used and/or analyzed during the current study are available from the corresponding author on reasonable request.

\section{Ethics approval and consent to participate}

The study was approved by the ethics committee of the Seventh Affiliated Hospital of Sun Yat-Sen University (approving number:2018SYSUSH-012), and the informed consent was signed by all included patients or their families.

\section{Consent for publication}

The signed cconsent for publication statement has been obtained from all patients and healthy volunteers.

\section{Competing interests}

The authors declare that they have no competing interests.

\section{Author details}

'Department of Urology, The Seventh Affiliated Hospital of Sun Yat-Sen University, Shenzhen 518107, China. ${ }^{2}$ Department of Clinical Laboratory, PLA 309 Hospital, Beijing, China. ${ }^{3}$ Department of Surgery Anesthesia Center, the Seventh Affiliated Hospital of Sun Yat-Sen University, Shenzhen 518107,

China.

Received: 5 November 2019 Accepted: 2 April 2020

Published online: 01 May 2020

\section{References}

1. Attard G, Parker C, Eeles RA, et al. Prostate cancer. Lancet. 2016;387:70-82.

2. Litwin MS, Tan HJ. The diagnosis and treatment of prostate cancer: a review. JAMA. 2017;317:2532-42.

3. Vlachostergios PJ, Puca L, Beltran H. Emerging variants of castration-resistant prostate cancer. Curr Oncol Rep. 2017;19:32.

4. Tugarova AV, Mamchenkova PV, Dyatlova YA, et al. FTIR and Raman spectroscopic studies of selenium nanoparticles synthesised by the bacterium Azospirillum thiophilum. Spectrochim Acta A Mol Biomol Spectrosc. 2018;192:458-63.

5. Winkler HC, Suter M, Naegeli H. Critical review of the safety assessment of nano-structured silica additives in food. J Nanobiotechnology. 2016;14:44.

6. Cruz LY, Wang D, Liu J. Biosynthesis of selenium nanoparticles, characterization and $\mathrm{X}$-ray induced radiotherapy for the treatment of lung cancer with interstitial lung disease. J Photochem Photobiol B. 2019;191: $123-7$.

7. Zhou Y, Xu M, Liu Y, Bai Y, Deng Y, Liu J, et al. Green synthesis of Se/Ru alloy nanoparticles using gallic acid and evaluation of theiranti-invasive effects in HeLa cells. Colloids Surf B Biointerfaces. 2016;144:118-24.

8. Wang $X$, Sun $K$, Tan Y, Wu S, Zhang J. Efficacy and safety of selenium nanoparticles administered intraperitoneally for the prevention of growth of cancer cells in the peritoneal cavity. Free Radic Biol Med. 2014;72:1-10.

9. Gao F, Yuan Q, Gao L, Cai P, Zhu H, Liu R, et al. Cytotoxicity and therapeutic effect of irinotecan combined with selenium nanoparticles. Biomaterials. 2014;35:8854-66.

10. Yao C, Li G, Cai M, et al. Prostate cancer downregulated SIRP-a modulates apoptosis and proliferation through p38-MAPK/NF-KB/COX-2 signaling. Oncol Lett. 2017;13:4995-5001.

11. Obhielo E, Ezeanochie M, Olokor OO, et al. The relationship between the serum level of selenium and cervical intraepithelial neoplasia: a comparative study in a population of Nigerian women. Asian Pac J Cancer Prev. 2019;20: 1433-6.

12. Chen S, Principessa L, Isaacs JT. Human prostate cancer initiating cells isolated directly from localized cancer do not form prostaspheres in primary culture. Prostate. 2012;72:1478-89.

13. Hinz M, Krappmann D, Eichten A, et al. NF-kappaB function in growth control: regulation of cyclin D1 expression and G0/G1-to-S-phase transition. 
NF- kappaB function in growth control: regulation of cyclin D1 expression and G0/G1-to-S-phase transition. Mol Cell Biol. 1999;19:2690-8.

14. Bao $H$, Zhang $\mathrm{Q}, \mathrm{Xu} \mathrm{H}$, et al. Effects of nanoparticle size on antitumor activity of 10-hydroxycamptothecin-conjugated gold nanoparticles: in vitro and in vivo studies. Int J Nanomedicine. 2016;11:929-40.

15. Xia Y, Chen Y, Hua L, et al. Functionalized selenium nanoparticles for targeted delivery of doxorubicin to improve non-small-cell lung cancer therapy. Int J Nanomedicine. 2018;13:6929-39.

16. Cui $D$, Liang $T$, Sun $L$, et al. Green synthesis of selenium nanoparticles with extract of hawthorn fruit induced HepG2 cells apoptosis. Pharm Biol. 2018; 56:528-34.

17. Vekariya KK, Kaur J, Tikoo K. ERa signaling imparts chemotherapeutic selectivity to selenium nanoparticles in breast cancer. Nanomedicine. 2012; 8:1125-32.

18. Sonkusre P, Cameotra SS. Biogenic selenium nanoparticles induce ROSmediated necroptosis in PC-3 cancer cells through TNF activation. J Nanobiotechnology. 2017;15:43.

19. Kong L, Yuan Q, Zhu H, et al. The suppression of prostate LNCaP cancer cells growth by Selenium nanoparticles through Akt/Mdm2/AR controlled apoptosis. Biomaterials. 2011;32:6515-22.

20. Liu C, Fu Y, Li CE, et al. Phycocyanin-functionalized selenium nanoparticles reverse palmitic acid-induced pancreatic $\beta$ cell apoptosis by enhancing cellular uptake and blocking reactive oxygen species (ROS)-mediated mitochondria dysfunction. J Agric Food Chem. 2017;65:4405-13.

21. Huang G, Liu Z, He L, et al. Autophagy is an important action mode for functionalized selenium nanoparticles to exhibit anti-colorectal cancer activity. Biomater Sci. 2018;6:2508-17.

22. Liu Z, Zhou G, Deng X, et al. Analysis of miRNA expression profiling in human macrophages responding to mycobacterium infection: induction of the immune regulator miR-146a. J Infect. 2014;68:553-61.

23. Bhattacharyya NP, Das E, Bucha S, et al. Regulation of cell cycle associated genes by microRNA and transcription factor. Microrna. 2016;5:180-200

24. Di Leva G, Garofalo M, Croce CM. MicroRNAs in cancer. Annu Rev Pathol. 2014;9:287-314.

25. Li Q, Zhang X, Li N, Liu Q. Chen D.miR-30b inhibits cancer cell growth, migration, and invasion by targeting homeobox A1 in esophageal cancer. Biochem Biophys Res Commun. 2017;485:506-12.

26. Liu F, Song D, Wu Y, Liu X, Zhu J, Tang Y. miR-155 inhibits proliferation and invasion by directly targeting PDCD4 in non-small cell lung cancer. Thorac Cancer. 2017;8:613-9.

27. Zhu Y, Xia Y, Niu H, et al. miR-16 induced the suppression of cell apoptosis while promote proliferation in esophageal squamous cell carcinoma. Cell Physiol Biochem. 2014;33:1340-8.

28. Cai CK, Zhao GY, Tian LY, et al. miR-15a and miR-16-1 downregulate CCND1 and induce apoptosis and cell cycle arrest in osteosarcoma. Oncol Rep. 2012:28:1764-70

29. Pekarsky Y, Balatti V, Croce CM. BCL2 and miR-15/16: from gene discovery to treatment. Cell Death Differ. 2018;25:21-6.

30. Shahverdi AR, Shahverdi F, Faghfuri E, Reza Khoshayand M, Mavandadnejad $\mathrm{F}$, Yazdi MH, et al. Characterization of folic acid surface-coated selenium nanoparticles and corresponding in vitro and in vivo effects against breast cancer. Arch Med Res. 2018;49:10-7.

31. Nazıroğlu M, Muhamad S, Pecze L. Nanoparticles as potential clinical therapeutic agents in Alzheimer's disease: focus on selenium nanoparticles. Expert Rev Clin Pharmacol. 2017;10:773-82.

\section{Publisher's Note}

Springer Nature remains neutral with regard to jurisdictional claims in published maps and institutional affiliations.

Ready to submit your research? Choose BMC and benefit from:

- fast, convenient online submission

- thorough peer review by experienced researchers in your field

- rapid publication on acceptance

- support for research data, including large and complex data types

- gold Open Access which fosters wider collaboration and increased citations

- maximum visibility for your research: over $100 \mathrm{M}$ website views per year

At $\mathrm{BMC}$, research is always in progress.

Learn more biomedcentral.com/submissions 